\title{
Colours + Numbers differs from colours of numbers: cognitive and visual illusions in grapheme-colour synaesthesia
}

\author{
Mariagrazia Ranzini ${ }^{1} \cdot$ Luisa Girelli ${ }^{2,3}$
}

Published online: 8 March 2019

(C) The Psychonomic Society, Inc. 2019

\begin{abstract}
This study investigates the bi-directionality of synaestesic experience by means of a flanked bisection paradigm in TT, a numbercolour synaesthete. Previous studies have shown that bisection is shifted towards the larger digit flanker (e.g., Ranzini \& Girelli, 2012). TT and controls performed line bisections with lines flanked by black digits (experiment 1), by TT's photism colours (experiment 2 ), and by congruently (experiment 3 ), or incongruently coloured digits (experiment 4 ). While the results of the control group mainly replicated previous findings, only the colour-digit congruence elicited in TT the larger-digit bias. TT's absence of effects in the other conditions was not due to reduced sensitivity to luminance effects (experiment 5), or to mathematical expertise (experiment 6). We suggest that grapheme-colour synaesthesia might be characterised by a rigid access to semantic representation when the inducer is task-irrelevant.
\end{abstract}

Keywords Numerical cognition $\cdot$ Synaesthesia $\cdot$ Line bisection $\cdot$ Magnitude processing

\section{Introduction}

Synaesthesia is a phenomenon in which one stimulated sensory modality leads to an experience in another nonstimulated stream (Galton, 1880). One case of synaesthesia consists of idiosyncratic associations between numbers and colours (number-colour synaesthesia). These synaesthetes report to have always perceived, or thought, specific numbers as associated with specific colours. This phenomenon sometimes extends to other graphemes, e.g., letters; for this reason this kind of synaesthesia is referred to as grapheme-colour synaesthesia. Grapheme-colour synaesthetes usually report that the colour "appears" to them each time a grapheme is evoked, whether thought, seen, or listened to. Grapheme-colour synaesthesia affects about the 1.1-1.4\% of the population (Simner et al., 2006), and between the $45 \%$ (Simner et al., 2006) and

Mariagrazia Ranzini

mari.ranzini@gmail.com; mariagrazia.ranzini@unipd.it

1 Department of General Psychology, University of Padova, via Venezia 8, 35131 Padua, Italy

2 Department of Psychology, University of Milano-Bicocca, Milan, Italy

3 NeuroMI, Milan Center for Neuroscience, Milan, Italy the $88 \%$ of synaesthetes (Sagiv, Simner, Collins, Butterworth, \& Ward, 2006; see also: Barnett et al., 2008; Day, 2005; Niccolai, Jennes, Stoerig, \& van Leeuwen, 2012; Rich, Bradshaw, \& Mattingley, 2005). A study by Niccolai et al. (2012) on a group of 63 synaesthetes, found that synesthesia for digits was more common (86\%) than other types of grapheme-colour synesthesia (e.g., for letters or words).

Despite high heterogeneity (i.e., number-colour associations vary across individuals), common features among the varieties of synaesthesia have been reported. First of all, synaesthetic associations are automatic perceptual experiences, as testified by results in the synaesthetic version of the Stroop task (Stroop, 1935), where stimuli (e.g., numbers) are presented in the congruent or incongruent colours for the particular synaesthete being tested (Wollen \& Ruggiero, 1983; but see Meier \& Rothen, 2009). Synaesthetes are usually faster responding to congruent stimuli compared to incongruent ones. Moreover, synaesthesia has been traditionally conceived as being unidirectional, that is, a stimulus (e.g., a digit) triggers the colour but not vice versa (e.g., Mills, Boteler, \& Oliver, 1999). However, increasing evidence has been provided about bi-directionality, showing that even colours can elicit experience of the corresponding associated items in synaesthetes (for a first review and discussion on bi-directional synaesthesia, see: Cohen Kadosh \& Henik, 2006). Among the different - sometimes rare - types of bi-directional synaesthesia (e.g., colours 
evoking sound experience: Goller, Otten, \& Ward, 2009), the most investigated one has been grapheme-colour synaesthesia (e.g., Brang, Edwards, Ramachandran, \& Coulson, 2008; Johnson, Jepma, \& De Jong, 2007; Weiss, Kalckert, \& Fink, 2009). In particular, bi-directional associations between number and colour have received large attention (e.g., Brugger, Knoch, Mohr, \& Gianotti, 2004; Cohen Kadosh \& Henik, 2006; Cohen Kadosh, Kadosh, \& Henik, 2007a; Cohen Kadosh et al., 2005; Gebuis, Nijboer, \& van der Smagt, 2009; Gevers, Imbo, Cohen Kadosh, Fias, \& Hartsuiker, 2010; Knoch, Gianotti, Mohr, \& Brugger, 2005; Teichmann, Nieuwenstein, \& Rich, 2015).

Importantly, for the most part, these studies have shown that in bi-directional synaesthethes the colour can trigger-explicitly or implicitly - not only the simple experience of a specific grapheme, but also the meaning of that grapheme, i.e., in the case of digits, the colour leads to access to the numerical magnitude of the digit. For instance, Cohen Kadosh and Henik (2006) reported a single-case study of grapheme-colour synaesthesia whose performance in a length comparison task was modulated by the colours of the lines corresponding either to larger or smaller numbers. This shows that synaesthetic colours could bind numerical and physical magnitude even in the absence of numbers. Importantly, synaesthetes often report to be unconscious of perceiving a number triggered by a colour, indicating that bi-directionality in synaesthesia can emerge without conscious awareness (Cohen Kadosh \& Henik, 2007; see also: Berteletti, Hubbard, \& Zorzi, 2010; Johnson et al., 2007).

The present study investigates the bi-directionality of number-colour associations in TT, a single case of grapheme-colour synaesthesia. TT reported automatically associating colours with numbers, so that "the colour is an attribute of the number", but not automatically activating the number when the colour is perceived. To investigate the presence of a bi-directional synaesthesia in TT, we used a cued line bisection task, with cues being numerical flankers or shades of TT's photism synaesthetic colours. The flanked line bisection has proved useful to explore the effects of numerical magnitude on spatial processing (Fischer, 2001; de Hevia, Girelli, \& Vallar, 2006): when participants bisect a horizontal segment flanked by two digits (e.g., 1-8 or 8-1), they misplace the midpoint towards the numerically larger digit, independent of its position (see also: Bonato, Priftis, Marenzi, \& Zorzi, 2008, in hemispatial neglect). De Hevia et al. (2006) interpreted this "larger-digit bias" as a cognitive illusion whereby magnitude processing results in an illusory compression (with small-magnitude numbers) or expansion (with large-magnitude numbers) of the perceptual space. Visual illusions would, thus, result from the influence of physical features of the stimulus on its perception (Baldwin, 1895), whereas cognitive illusions would result from the influence of semantic information (e.g., magnitude) on perception. This effect testifies to an implicit access to numerical magnitude even in number-irrelevant task.
Furthermore, Ranzini and Girelli (2012) showed that luminance modulates bisection performance by shifting the subjective midpoint toward the darker flanker, and that when luminance and numbers were combined, by displaying numerical flankers in shades of grey, luminance reduced the larger-digit bias when incongruently combined with the numerical information (i.e., when smaller and larger digits were presented in darker and brighter shades of grey, respectively). Importantly, the association between small/large numbers and bright/dark luminance in nonsynaesthetes is similar to the tendency to associate darker colours (i.e., less luminance) to larger numbers as established in synaesthetes (Beeli, Esslen, \& Janke, 2007; Cohen Kadosh, Henik, \& Walsh, 2007b; Smilek, Carriere, Dixon, \& Merikle, 2007).

In this study we examined to what extent TT's bisection performance was modulated by his number-colour associations. If TT's synaesthesia is bidirectional, we would expect TT's performance to be modulated by the numerical magnitude per se, i.e., black numbers (experiment 1), by TT's photism colour (experiment 2), and by the combination of the two (whether combined congruently or incongruently, experiments 3-4). Finally, we also verified that TT's performance in these tasks was not due to a low sensitivity to luminance (experiment 5) or to his level of mathematical expertise (experiment 6), rather than to synaesthesia itself.

\section{General method}

\section{Participants}

TT At the time of testing TT was a 27 year-old, male, $\mathrm{PhD}$ student in maths. He was originally recruited as a volunteer participant in a study on number processing in maths experts and, at that time, he was completely unaware of his synaesthesia. As a standard testing routine, we asked him whether he had any form of synaesthesia, and he was really shocked when realizing that his associations were not universally shared. We first assessed TT's synaesthesia with a questionnaire (Hubbard, Ranzini, Piazza, Dehaene, 2009), which confirmed his grapheme-colour synaesthesia.

To test the automaticity of TT's digit-colour associations, we used a version of the synaesthetic Stroop task, a task commonly used to evaluate grapheme-colour associations in synaesthesia (Wollen \& Ruggiero, 1983). We firstly asked to TT to edit his synaesthetic colours corresponding to the digits from 1 to 9 by using the colour mixer tool in Microsoft Paint. We then encoded TT's colours as RGB colour codes, and in a separate session we presented him with each digit coloured in his synaesthetic colour. In this session, the digits were presented one by one in Courier New 27 (the same font and size were used for stimuli in the Stroop task and in all 
other experiments). TT was required to indicate whether the colours on the screen closely matched his synaesthetic experience. For each digit, we also asked TT to name the colour. He named the colour corresponding to the digits 1,2,8, and 9, respectively, "grey", "yellow", "burgundy", "black" (in Italian: "grigio", "giallo", "bordeaux", "nero"). TT used these terms to name the colours in the Stroop task. The Stroop task was composed of 144 trials ( 3 blocks of 48 trials each) where the digits $1,2,8$, or 9 were presented in a congruent condition (i.e., in the synaesthetic colour, e.g., 1 as "grey") or in an incongruent condition (i.e., in an incongruent colour: 1 as "yellow" or "burgundy"). The incongruent combinations, in all bisection experiments were the following: 1 coloured as 2 or $8 ; 2$ coloured as 1 or $9 ; 8$ coloured as 1 or $9 ; 9$ coloured as 2 or 8 . Thus, half of the trials were congruent, and half of the trials were incongruent to TT's synaesthetic associations. The trial started with a fixation point lasting $500 \mathrm{~ms}$, followed by a blank screen lasting 500ms. Then the stimulus appeared and it remained on the screen until a response was given. TT was required to name as fast as possible the colour of the digit. Response times (RTs) were recorded by the use of a microphone, while the responses were manually encoded by the experimenter, which was standing out of TT's sight. After an inter-trial-interval (ITI) lasting $500 \mathrm{~ms}$, the next stimulus appeared. The stimuli were presented on a white background. Microphone errors (1\%), incorrect responses (less than $1 \%$ ), and RTs outliers (over \pm 2.5 SD: $2 \%$ ) were excluded from the analysis. TT named the digit colour when the colour-digit mapping matched his synaesthetic association faster $(\mathrm{M}=681 \mathrm{~ms}, \mathrm{SE}=12.1)$ than when the digit colour corresponded to another digit in his associations $(M=757 \mathrm{~ms}$, $\mathrm{SE}=17.6$; test $\mathrm{t}$ considering the mean RT for each stimulus as independent observation: $\mathrm{t}(10)=-3.47, \mathrm{p}<.01)$. To take into account the numerical distance between the presented digit and the digit corresponding to the associated colour, we ran an ANOVA with Condition (Congruent, Incongruent Close, Incongruent Far) as factor. In the incongruent close condition, the digits were paired with colours associated by TT to close digits (e.g., a yellow 1). In the incongruent far condition, the digits were paired with colours associated by TT to far digits (e.g., a burgundy 1). The effect of Condition was significant $(\mathrm{F}(2,9)=6.044, \mathrm{p}<.05)$ : congruent stimuli were answered faster than incongruent close ones ( $\mathrm{M}$ for incongruent close stimuli $=767 \mathrm{~ms}$, post-hoc comparison: $\mathrm{p}<.05$ ). Post-hoc comparisons revealed no significant difference between congruent and incongruent far stimuli ( $\mathrm{M}$ for incongruent far stimuli = $747 \mathrm{~ms}, \mathrm{p}<.07$ ), and between incongruent far stimuli and incongruent close ones ( $p>$.1). TT's number-colour associations are represented in Figure 1.

The control group in baseline bisection and experiments 1-4 Eight non-synaesthete participants (mean age $=28$, range $=23$ 33) matched for sex and handedness to TT served as controls

\section{$\begin{array}{lllllll}3 & 4 & 5 & 6 & 7 & 8 & 9\end{array}$}

Fig. 1 TT's number-colour associations. The illustration indicates for each digit TT's synaesthetic colour experience (see online version for color figure)

in experiments 1-4. All were naive with regards to the hypotheses of the study.

\section{Materials and experimental procedure}

The task was a computerized line bisection: participants indicated the line midpoint with the help of the mouse (see Figure 2 for details).

Baseline bisection TT was first compared to the controls in a computerized baseline bisection (10 training +48 experimental trials). The experiment lasted about 5 minutes.

Experiments 1-4 In experiments 1-4, task-irrelevant flankers were presented at line extremes (Figure 3). The flankers were black digits (experiment 1), TT's photism colours (experiment 2 ), and digits coloured congruently (experiment 3 ) or incongruently (experiment 4) with respect to TT's digit-colour mapping. Note that smaller digits were brighter than larger digits in TT's synaesthesia (Figure 1), therefore congruent and incongruent digits with respect to TT's digit-colour mapping were also congruent and incongruent with respect to controls, for which associations between small/large numbers and bright/dark luminance has been shown (Ranzini \& Girelli, 2012). The pair of numerical flankers had different numerical distances (close: $1 / 2$ or 8/9; far: 1/8 or 2/9). The position of the larger number flanker (left/right) was counterbalanced. Each experiment lasted about 10 minutes (10 practice +96 experimental trials).

\section{Scoring and statistical analysis}

At each trial the participant indicated the subjective midpoint by using a mouse device. The mouse cursor, visible to the participant, corresponded to an upward-pointing arrow (see Figure 1). The extremity of the arrow head was one pixel wide and pointed to a single pixel located under the line. Each participant's response corresponded to the arrow head location (arrow head horizontal coordinate on the screen) when the participant clicked the left mouse button. This method can reliably assess individual variability at the level of 1 pixel (see also: Arduino, Previtali, \& Girelli, 2010; Perrone, de Hevia, Bricolo, \& Girelli, 2010; Ranzini \& Girelli, 2012). Accuracy (in pixels) was calculated by subtracting the real midpoint from the subjective midpoint: positive values corresponded to rightward biases, and negative values to leftward biases. To compare TT's performance to the control group in the baseline bisection, a statistical procedure adapted for comparing an individual's test score against norms derived 


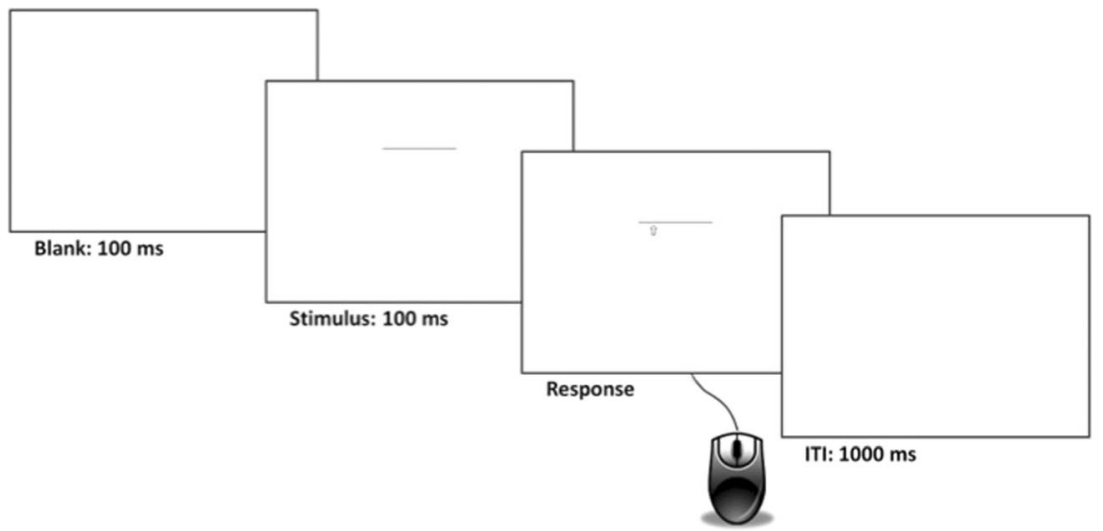

Fig. 2 Example of a trial in the baseline bisection task. The mouse cursor was a vertical arrow that appeared either under the left or under the right extreme of the line, at a fixed distance of five pixels under the stimulus. The participant could move the arrow cursor only horizontally, leftward or rightward, under the line to choose the line midpoint. To increase stimulus variability, two different line lengths were presented (shorter: 236 pixels; longer: 315 pixels, height: 4 pixels), and each line was presented slightly shifted from the screen centre (six different positions: up/centre/down + leftward/rightward, misplaced 77 pixels up or down, and 102 pixels right or left from the centre). Line length was

\section{Exp. $1 / 6$}

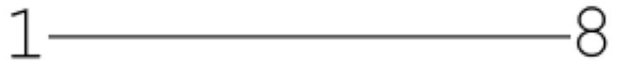

Exp. 2

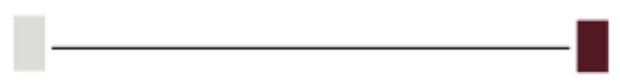

Exp. 3

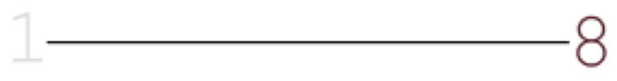

Exp. 4

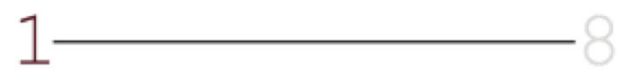

\section{Exp. 5}

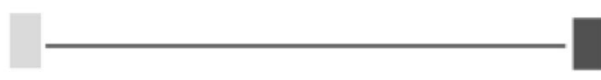

Fig. 3 Example of the stimuli for each experiment. The flankers were presented at a distance of $4 \mathrm{px}$ from the line. The numbers were black digits (Courier New 27) in experiment 1 and 6, rectangles (size: $16 \times 22$ pixel) of TT's photism colours in experiment 2, digits coloured in TT's congruent colours in experiment 3 , and digits coloured in TT's incongruent colours in experiment 4 , and rectangles of different shades of grey in experiment 5. Controls performed the experiments 1-4 in a single session. TT performed experiment 1 , experiments $2-4$, and experiment 6 in separate sessions. The order of the experiments 1-4 was the same for TT and controls (experiments 1,2,4,3) (see online version for color figure) counterbalanced, while the initial position of the mouse cursor and the position of the line on the screen were randomized. Line length, line position, and the mouse cursor initial position were not analyzed because they did not constitute experimental manipulations. The stimuli were presented on a portable DELL pc (screen resolution: 1024x768). The participants were instructed to keep a central position at a distance of about $60 \mathrm{~cm}$ from the screen. There was no time limit, although the instructions emphasized both speed and accuracy. The baseline bisection task was modified by the presence of task-irrelevant flankers at both line extremes in experiments 1-6 (Fig. 3)

from small samples was used at two-tailed significance level for the experiments 1-4 (Crawford \& Howell, 1998). For the experiments 5-6, one-tailed significance level was used since we had a-priori predictions, based on results of the experiments 1-4, about the direction of the effects. For the control group, experiments 1-4 were analysed with repeated measures ANOVAs with Position Larger (larger-digit left (LL)/largerdigit right (LR)) and Numerical Distance (close/far) as withinsubject factors. The revised standardized difference test (RSDT, Crawford \& Garthwaite, 2005) was then used to test whether the difference between TT's performance in the two Position Larger levels was significantly different from that observed in the controls. In each experiment, we also compared TT's and controls' median RTs (for each trial, the RT corresponded to the amount of time from the onset of the stimulus to the response) to rule out the possibility that the results observed on the bisection bias could be attributed to speed of processing differences.

\section{Experiment 1-4 and baseline bisection: results}

A summary of TT's and of the controls' mean bias for the two conditions relative to the flankers' position is given in Table 1 (bias expressed in pixel) and Table 2 (bias expressed as percentage of line length). Across all experiments the pattern of results was identical when performing the analyses on the bias expressed as percentage of line length and on the bias expressed in pixel. To facilitate the comparison between the present results and the results of our previous study (Ranzini 
Table 1 TT's and the controls' mean bias (M), and the standard deviation (SD) of the control group are reported, expressed in pixels, for the baseline bisection (BB) and for the two conditions relative to the flankers' position in experiments 1-6

\begin{tabular}{|c|c|c|c|c|}
\hline & \multicolumn{2}{|l|}{ TT: $M_{-}$} & \multicolumn{2}{|c|}{ Control Group: $M(S D)$} \\
\hline & $\mathrm{LL} / \mathrm{DL}$ & $\mathrm{LR} / \mathrm{DR}$ & LL / DL & $\mathrm{LR} / \mathrm{DR}$ \\
\hline BB & 3.7 & & $1.9(3.5)$ & \\
\hline Experiment 1 & 3.7 & 3.5 & $0.6(2.9)$ & $2.0(2.9)$ \\
\hline Experiment 2 & 3.0 & 2.2 & $1.2(3.0)$ & $2.0(2.3)$ \\
\hline Experiment 3 & 1.7 & 3.2 & $0.0(3.4)$ & $2.6(2.8)$ \\
\hline Experiment 3: retest & 3.2 & 4.7 & & \\
\hline Experiment 4 & 3.4 & 3.1 & $1.0(3.4)$ & $0.8(3.5)$ \\
\hline Experiment 5 & 3.9 & 4.1 & $-1.0(2.9)$ & $-0.5(2.4)$ \\
\hline Experiment 6 & & & $-1.1(1.6)$ & $-0.2(1.7)$ \\
\hline
\end{tabular}

Note. LL = Larger Left; LR = Larger Right; DL = Darker Left; DR = Darker Right. LL and LR refer to the experimental conditions in experiments 1-4 and 6, while DL and DR refer to the experimental conditions in experiment 5

\& Girelli, 2012), we report here the analyses based on the bias expressed in pixel.

\section{Baseline bisection}

The controls' bisection performance $(M=1.9 \mathrm{px}, S D=3.5)$ did not differ from accurate bisection $(t(7)$ vs. $0=1.5, p>.1)$. Importantly, TT's performance (3.7px) did not significantly differ from that of the controls $(p>.6)$. We also compared TT's and controls' median RTs. TT was as fast $(M d n=1960 \mathrm{~ms})$ as the controls (mean and standard deviation of median RTs: $M=1945 \mathrm{~ms}, S D=1286 ; p>.9)$.

\section{Experiments 1-4}

The effects of the flankers position in bisection performance in TT and the controls (experiments 1-6) are illustrated in Figure 4.
Experiment 1 In the controls a significant main effect of Position Larger was observed (LL: $M=0.6, S D=2.9$; LR: $M=2.0, S D=2.9 ; F(1,7)=81.07, p<.001)$, modulated by Numerical Distance $(F(1,7)=7.65, p<.05)$ : the large digit bias emerged in far distance (LL: $M=-0.2, S D=3.0$; LR: $M=2.3$, $S D=2.7 ; t(7)=-4.80, p<.005$ ) but not in close one (LL: $M=1.4, S D=3.0$; LR: $M=1.6, S D=3.2 ; p>.6$ ). The main effect of Numerical Distance was not significant. TT's performance (LL: $M=3.7$; LR: $M=3.5$ ) differed from that of the controls $(t(7)=2.82, p<.05)$, indicating no such effect of Position Larger in TT.

Experiment 2 An effect of Position Larger (i.e., in this case the colour corresponding to a larger digit for TT) characterized the controls' performance $(F(1,7)=12.16, p<.05)$ (LL: $M=1.1$, $S D=3.0$; LR: $M=2.0, S D=2.3)$. No other main effects or interactions were significant. TT's Position Larger effect again

Table 2 TT's and the controls' mean bias (M), and the standard deviation (SD) of the control group are reported, expressed in percentage of line length, for the baseline bisection (BB) and for the two conditions relative to the flankers' position in experiments 1-6

\begin{tabular}{|c|c|c|c|c|}
\hline & \multicolumn{2}{|l|}{ TT: $M_{-}$} & \multicolumn{2}{|c|}{ Control Group: $\mathrm{M}(S D)$} \\
\hline & $\mathrm{LL} / \mathrm{DL}$ & $\mathrm{LR} / \mathrm{DR}$ & $\mathrm{LL} / \mathrm{DL}$ & $\mathrm{LR} / \mathrm{DR}$ \\
\hline $\mathrm{BB}$ & 1.29 & & $0.66(1.3)$ & \\
\hline Experiment 1 & 1.33 & 1.29 & $0.20(1.0)$ & $0.72(1.1)$ \\
\hline Experiment 2 & 1.08 & 0.78 & $0.37(1.1)$ & $0.72(0.8)$ \\
\hline Experiment 3 & 0.57 & 1.08 & $0.00(1.2)$ & $0.94(1.0)$ \\
\hline Experiment 3: retest & 1.11 & 1.71 & & \\
\hline Experiment 4 & 1.20 & 1.04 & $0.37(1.3)$ & $0.28(1.3)$ \\
\hline Experiment 5 & 1.47 & 1.49 & $-0.36(1.0)$ & $-0.17(0.9)$ \\
\hline Experiment 6 & & & $-0.41(0.6)$ & $-0.05(0.6)$ \\
\hline
\end{tabular}

Note. $\mathrm{LL}=$ Larger Left; LR = Larger Right; DL = Darker Left; DR = Darker Right. LL and LR refer to the experimental conditions in experiments 1-4 and 6, while DL and DR refer to the experimental conditions in experiment 5 

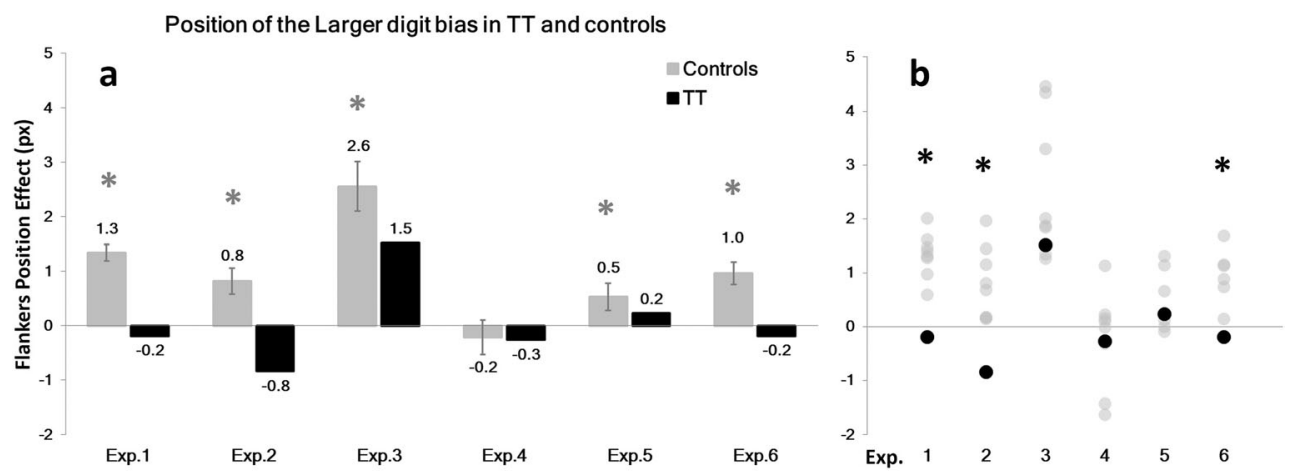

Fig. 4 Illustration of the main results. a) To illustrate the different effects of the flankers position in bisection performance between TT and the controls, we plotted the larger digit - right minus left difference (experiments 1, 3, 4, and 6), the difference between TT's photism colours conditions (experiment 2), and the darker flanker - right minus left difference (experiment 5). In this way, a positive value corresponded to a bisection bias toward the larger digit (experiments 1, 3, 4, and 6),

toward TT's photism colour (experiment 2), or toward the darker flanker (experiment 5). Error bars indicate SEM, and asterisks indicate for each experiment a significant effect of flankers position in the group of control (see the Results sections, for details). b) Plot of the flankers position effect for TT and each control participant. Asterisks indicate for each experiment a significant difference between TT and the controls (see the Results sections, for details)

differed from that of the controls $(t(7)=5.72, p<.001$; TT: LL $M=3.0$; LR $M=2.1$ ). Overall these results suggest that the controls were sensitive to colours as they tended to misperceive the line midpoint towards the darker colour: indeed, larger digits correspond to darker colours in TT's associations (see Figure 1). Interestingly, TT did not show this effect.

Experiment 3 Position Larger in controls was again significant (LL: $M=0.0, S D=3.4$; LR: $M=2.6, S D=2.8 ; F(1,7)=31.19$, $p<.001)$. This effect interacted with Numerical Distance $(F(1,7)=28.63, p<0.005)$ : the larger-digit bias was limited to far distance (far distance: LL $M=-0.7, S D=2.9$; LR $M=3.3$, $S D=2.8 ; t(7)=-8.42, p<.001$; close distance: LL $M=0.8$, $S D=4.0$; LR $M=1.8, S D=3.0 ; p>.1)$. Numerical Distance was not significant. TT's performance did not differ from the controls, either overall (LL: $M=1.6$; LR: $M=3.2$ ) or for separate distances (far distance: LL $M=0.9$, LR $M=4.6$; close distance: LL $M=2.4$, LR $M=1.7$; all $p>.3$ ), indicating that now the Position Larger bias was similar in both the controls and TT. To verify the consistency of this effect, TT was retested in a separate session. TT's performance again did not differ from that of the controls either overall (LL: $M=3.2$; LR: $M=4.7$ ) or for separate distances (far distance: LL $M=2.5$, LR $M=6.1$; close distance: LL $M=3.8$, LR $M=3.3$; all $p>.5$ ).

Experiment 4 Controls' performance was not affected by any experimental factors (all $p>.5$ ), and TT's performance did not differ from that of the controls in the larger-digit bias, indicating that no Position Larger bias was present in TT (LL $M=3.4$, LR $M=3.1$ ) or in the control group (LL: $M=1.0, S D=3.4$; LR: $M=0.8, S D=3.5$; TT vs. controls: $p>.9)$.

\section{RTs analyses for experiments 1-4}

We compared TT's and controls' median RTs for each experiment. TT was as fast as controls in all experiments (all $p>.5$; TT's median RTs: $2839 \mathrm{~ms}, 1928 \mathrm{~ms}, 1733 \mathrm{~ms}, 1484 \mathrm{~ms}$, for experiments 1-4 respectively; controls' median RTs for experiment 1: $M=2026 \mathrm{~ms}, S D=1317$; experiment 2: $M=1974 \mathrm{~ms}$, $S D=1316$; experiment 3: $M=1931 \mathrm{~ms}, S D=1236$; experiment 4: $M=1788 \mathrm{~ms}, S D=934)$. A repeated-measures ANOVA on median RTs of the controls with Experiment (Arduino et al., 2010; Arend et al., 2016; Baldwin, 1895; Banissy et al., 2013) as within-subject factor indicated no significant main effect of Experiment $(p=.35)$. Taken together, these results suggest that TT behaved similarly in all experiments and that the differences observed with regards to the larger-digit effect across experiments cannot be attributed to different execution strategies. However, the fact that TT was slower in experiment 1 as compared to all other experiments might reflect an order effect, since experiment 1 was the first experiment to be conducted.

\section{Experiments 1-4: Discussion}

While the controls showed the larger-digit effect with black digits (experiment 1) and with TT's congruently coloured digits (experiment 3 ), TT showed this effect only with congruent coloured digits (experiment 3). When only TT's photism colours were presented (experiment 2), TT failed to present the effect observed in the controls', for which performance was modulated by the luminance disparities between flankers. Given that the brightness of TT's colours for digits co-varies with numerical magnitude - i.e. smaller numbers are brighter than larger ones - we explain this effect as a visual illusion 
according to which the subjective midpoint is shifted toward the darker flanker (Ranzini \& Girelli, 2012). TT's performance did not differ from that of the controls in experiments 3 and 4, showing that congruently coloured digits act for him as numerical cues (experiment 3), while incongruently coloured digits do not (experiment 4). Importantly, TT's performance was not modulated by digits or by colours as it was for the controls when they were presented separately (experiment 1-2). This pattern of results suggests that in colourgrapheme synaesthesia the association of colour and digit is critical to allow numbers to activate their semantic representation when irrelevant to the task at hand.

To confirm this conclusion, we first compared TT's and controls' performances in a grey flanked bisection task (experiment 5, Figures 2-3), to test whether TT's low sensitivity to luminance, rather than synaesthesia itself, could have been responsible for the lack of effect in experiment 2 . We then compared TT's bisection of lines flanked by black digits to a control group with similar educational background in mathematics (experiment 6), to test whether mathematical expertise, rather than synaesthesia itself, could have been responsible for the lack of effect in experiment 1.

\section{Experiment 5: Method}

The task and procedure were the same as for experiment 2, but here the flankers were rectangles filled in by different shades of grey (see Ranzini \& Girelli, 2012, for details).

\section{Participants}

TT and six non-synaesthetes controls matched for sex and handedness (mean age $=24$; range $=20-28$ ) participated to the experiment.

\section{Experiment 5: Results}

The average scores were submitted to a T-test comparing the two levels of the Position Darker (darker flanker left (DL)/ darker flanker right (DR)). An effect of Position Darker was present in the control group (DL: $M=-1.0 ; S D=2.9$; DR: $M=-$ $0.5, S D=2.4 ; t(5)=-2.14, p<.05)$, and TT's performance (DL: $M=3.9$; DR: $M=4.1)$ did not differ from that of the controls $(p>$.2). We also compared TT's and controls' median RTs. TT was as fast $(M d n=1948 \mathrm{~ms})$ as controls (mean and standard deviation of median RTs: $M=2014 \mathrm{~ms}, S D=651)$ in answering $(p>9)$.

The lack of significant difference between TT and controls in this experiment might be due to the fact that the shades of grey we used were not as effective as the colours used in experiment 2 , therefore minimizing the effect of the darker flanker in the control group. We ruled out this alternative explanation by comparing with an independent samples Ttest the size of the effect (LR minus LL) of the controls in experiment 2 and the size of the effect in experiment 5 . The effect was larger in experiment $2(M=0.8 \mathrm{px})$ than in experiment $5(M=0.5 \mathrm{px})$, but the difference was not significant ( $p>$.2), indicating that TT's sensitivity to luminance was within the normal range.

\section{Experiment 6: Method}

The method and procedure were identical to those in experiment 1 .

\section{Participants}

The control group was composed of six non-synaesthetes controls matched for sex, handedness (mean age $=28, S D=4.6$ ), and with a similar strong educational background in mathematic ( $\mathrm{PhD}$ students or $\mathrm{PhD}$ in mathematics or physics). As for TT, these controls were originally recruited as volunteers in a study on the effects of mathematical expertise on number processing. For that study, they were required to perform several tasks assessing their level of numerical and mathematical expertise (e.g., arithmetic facts, multi-digit computation, numerosity estimation, number comparison, parity judgment). The performance of the group of expert participants was always significantly better compared to the performance of a group of students from faculties not including an intensive training in mathematics (Ranzini, 2010).

\section{Experiment 6: Results}

The average scores were submitted to a test $t$ comparing the two levels of the Position of the Larger Digit (larger-left (LL)/ larger-right (LR)). The controls showed the Position Larger bias (LL: $M=-1.1, S D=1.6$; LR: $M=-0.2, S D=1.7 ; t(5)=-4.5$, $p<.01$ ). TT's performance (from experiment 1: LL: $M=3.7$; LR: $M=3.5)$ again differed from that of the controls $(t(5)=2.07 ; p<.05)$. We also compared TT's and controls' median RTs. TT was as fast (from experiment $1: M d n=2839 \mathrm{~ms}$ ) as controls (mean and standard deviation of median RTs: $M=4392 \mathrm{~ms}, S D=1563$ ) in answering ( $p>4)$.

\section{Experiments 5-6: Discussion}

Experiments 5 and 6 showed that TT's lack of a significant larger-digit bias was neither due to a reduced sensitivity to luminance (experiment 5), or to his mathematical expertise (experiment 6). Therefore, TT's results in experiments 1-4, 
i.e. being sensitive to numerical information only when numbers are presented with his colour association, should be safely attributed to his synaesthesia.

\section{General discussion}

We investigated the bi-directionality of number-colour associations in TT, a single case of grapheme-colour synaesthesia. To this aim we exploited the cued line bisection task and, specifically, the larger-digit bias that consists of a systematic misplacement of the subjective midpoint towards the larger of two flanker digits. This effect appears to reflect an interaction between visuospatial and numerical magnitude processes acting at a semantic level (de Hevia et al., 2006). After testing TT's average performance in a baseline bisection task, TT and controls performed four versions of the cued line bisection task, where flankers were black digits (experiment 1), TT's photism colours (experiment 2), and congruently (Experiment 3 ) or incongruently (experiment 4) coloured digits. We were interested in investigating whether TT would show illusory effects modulated by his synaesthesia.

The controls' performance was modulated by both numerical and colour information, as well as their combination. First, the control group showed a systematic misplacement of the subjective midpoint towards the larger flanker digit, both when flankers were black digits (experiment 1 ) and when they were congruently coloured with TT's synaesthetic associations (experiment 3). Moreover, in the controls, the coloured flankers induced a shift of the subjective centre towards the darker colour (experiment 2, corresponding to TT's larger digit), confirming previous findings (Ranzini \& Girelli, 2012). Critically, when numbers and colours luminance were incongruently combined, i.e. small digits were matched with dark colours and vice versa, no systematic bias was observed in the controls (experiment 4). On the other hand, TT showed a larger-digit bias only when the numerical flankers were coloured according to his synaesthetic associations (experiment 3 ) and, as for the controls, this effect disappeared with incongruently coloured digits (experiment 4). Critically, with black digits (experiment 1) or TT's photism colours (experiment 2) TT's performance differed from that of the controls, suggesting that cognitive and visual illusions per se did not modulate TT's bisection. TT's absence of effects was not due to his mathematical expertise (experiment 6 ) or to reduced sensitivity to illusory effects of luminance (experiment 5). Therefore, this pattern of results may suggest that magnitude in TT was processed only when fully matching his synaesthetic representation, i.e., when the flanker digit is correctly coloured with respect to his synaesthetic experience.

It is important to note that neither black digits (experiment 1) nor synaesthetic colours (experiment 2) elicited the larger digit effect in TT, which was instead observed for the controls in both experiments. Yet, the lack of effect when digits and colours were incongruently combined might have different origins for TT and for the controls. In TT this lack of effect can result from no effect for either colour or digit, while for the controls it is more likely to result from the interactive effects of colour luminance and digit (see also: Ranzini \& Girelli, 2012). In the control group, the effect of colour per se (experiment 2), as well as the interactions between colour and numerical magnitude (experiment 3 and 4), plausibly mirrors the effect induced by different degrees of luminance (i.e., brighter or darker colours), instead of a sort of synaesthetic experience. Indeed, the pattern of results observed in the controls closely mirrors the results of our previous study (Ranzini \& Girelli, 2012), where numerical flankers varying in luminance yielded a reduced larger-digit bias when the larger digit was brighter than the smaller one.

While in our previous study the effect of the larger digit was also present (albeit reduced) with incompatible mapping of luminance and numerical magnitude (i.e., larger and smaller digits were presented brighter and darker, respectively), here we observed no such effect in that condition (experiment 4). Several factors might have contributed to this discrepancy, such as interindividual variability (see Figure 4, panel b), or again the fact that the colours used in the present study might be somehow more effective than shades of grey in opposing the effect of digit (note that, the comparison between experiment 2 and 5, does not support this hypothesis). Again, a closer look to the size of the flankers bias shown by the controls in experiments 1 and 2 suggests that luminance and numerical magnitude might not interact additively: indeed, the size of the bias when colours and digits are combined (experiments 3 and 4) did not correspond exactly to the sum, or difference, of the size of the bias shown when digits (experiment 1 ) or colours (experiment 2) were presented in isolation. Importantly, in our previous study (Ranzini \& Girelli 2012) we also found evidence for the hypothesis that luminance and numerical magnitude do not interact additively. Overall, the results from the controls of this study confirmed that both numbers and luminance affect line bisection, and that luminance and numerical magnitude interact, possibly in a nonadditive manner (see also: Pinel, Piazza, Le Bihan, \& Dehaene, 2004).

Surprisingly, TT's performance is beyond bi-directional synaesthesia, and it mostly fits with evidence for a reduced flexibility in accessing numerical information in numbersrelated synaesthesia (Gertner, Henik, \& Cohen Kadosh, 2009). Gertner et al. (2009) reported three number-form synaesthethes (a variant of synaesthesia consisting of perceiving a numerical sequence as spatially oriented: e.g., Eagleman, 2009; Hubbard et al., 2009; Piazza, Pinel, \& Dehaene, 2006; Sagiv et al., 2006), whose number comparison performance was facilitated when numbers were presented in a spatial layout compatible with their synaesthetic number line. 
Interestingly, besides a congruency effect in number-form synaesthesia, they found that when digits where presented in a congruent layout, the distance effect (faster responses to numerically far numbers than to close ones) characterized synaesthetes' performance. Since the distance effect is universally considered a signature of semantic processing, these results suggest that access to semantic representation occurs only when stimuli presentation is in accordance with the synaesthetic experience. TT's results strengthen this view since numerical magnitude biased his performance only when there was explicit correspondence between the digit and its synaesthetic colour. In other words, only the correct digitcolour combination made irrelevant numbers fully meaningful.

This finding suggests that, under specific task conditions, i.e., when graphemes are task-irrelevant, only the meaning of stimuli (i.e. numerical magnitude) fully corresponding to the synaesthete's mental representation is reached. At a first sight, TT's performance in the Stroop task might seem to contradict this conclusion, if we consider that a slower response in naming the colour of an incongruent stimulus indicates that the task-irrelevant digit has been processed. However, we might be cautious in comparing results from the Stroop and the flanker tasks. Indeed, in the numerical Stroop task one dimension of the stimulus, in this case the colour, is task-relevant, while in the flanker task neither the digit nor the colour are task-relevant. Moreover, in the cued line bisection the flanker is physically separated from the line, while in the Stroop task the relevant and the irrelevant information belong to the same stimulus. This might trigger different processes in the Stroop and the flanker tasks. As suggested by previous studies, performance in the Stroop task is expected to be due to interference between two magnitude information, since facilitation for congruent stimuli is not likely to occur (e.g., see Dixon, Smilek, Cudahy, \& Merikle, 2005; Mills, Boteler, \& Oliver, 1999; MacLeod, \& MacDonald, 2000). The interference effect in incongruent stimuli suggests that two magnitudes are accessed, i.e. the magnitude of the digit and the magnitude associated to the colour, and slower response times might reflect the cost due to inhibit the processing of irrelevant information. On the contrary, TT's performance in line bisection with incongruent flankers (experiment 4) cannot be explained as the effect of interference between two magnitudes, since response times did not differ between conditions. Importantly, the larger-digit bias did not occur when flankers were either black digits (experiment 1 ) or synaesthetic colours (experiment 2), suggesting that, when presented one a time, irrelevant information was not fully processed and no magnitude was accessed. Similarly, TT might have not fully processed incongruent flankers in experiment 4 , since neither the digit nor the colour per se, induced the larger-digit/darker-colour bias. Taken together, the results suggest that in the flanker task the irrelevant digit or colour is not enough to access number magnitude except when paired with TT's synaesthetic experience (experiment 3 ).

Previous studies investigating bi-directionality in numbercolour synaesthesia mostly adopted tasks where at least one of the two dimensions (number or colour) was relevant (e.g., numerical Stroop: Cohen Kadosh et al., 2005; number/ colour detection with colour/number priming: Gebuis et al., 2009; mathematical problems verification: Gevers, et al., 2010, McCarthy et al., 2013; random color generation: Knoch et al., 2005), as well as physical magnitude tasks (Cohen Kadosh, \& Henik, 2006). Recently, Niessen, Fink, Schweitzer, Kluender, and Weiss (2015) provided evidence in favor of the idea that bi-directional synaesthesia occurs also when only the colour is presented and is irrelevant to the task. In their study, number-colour synaesthetes were required to bisect lines coloured with colours that the synaesthetes associated to specific numbers. In line with the assumption of a left-to-right mental number representation (Dehaene, Bossini, \& Giraux, 1993), synaesthetes misperceived the centre of the line towards the left when the line colour corresponded to a small number, compared to when the line colour corresponded to a large number. As for the Stroop task, this finding apparently contradicts our view that a limited access to numerical magnitude occurs when the synaesthetic stimulus is task-irrelevant. However, again, other factors may account for this difference. For instance, in Niessen et al.'s study (2015) the line and the colour were part of the same stimulus, while in the present study the line was visually separated from the flankers. It will be important for future studies to determine the degree of task-relevant information required to elicit bi-directional synaesthetic experiences.

Signatures of semantic processing (the larger-digit effect) emerged in TT only if the irrelevant numbers were combined congruently with TT's photism colours, and this suggests that, in all other cases, no magnitude was triggered by the flankers. Note that we are not claiming that TT did not process at all the flankers when digit and colour were not congruently combined, instead we suggest that the synaesthetic information, when not fully compatible and task-irrelevant, was not enough to reach the magnitude dimension. These results are in line with the idea that in number-colour synaesthesia, colour and digit are both parts of one same concept, and we make the hypothesis that, at some level of processing, the colour and the digit are not two dimensions bound together, but are together belonging to the same dimension. A number of studies have provided evidence for the idea that, in grapheme-colour synaesthetes, colour and grapheme can be both parts of the same concept. For instance, Meier and Rothen (2007) have conditioned in a group of grapheme-colour synaesthetes a startle response to a colour associated to a specific grapheme. After conditioning, a startle in response to the grapheme corresponding to the conditioned colour was observed in the synaesthetes (see also Rothen, Nyffeler, von Wartburg, Müri, 
\& Meier, 2010). Importantly, the idea that only the correct digit-colour combination induced in TT allows access to number semantic matches TT's self-report: as he claimed several times during informal discussions about his synaesthesia, in TT's world the colour is an attribute of the number. In other words, for TT, numbers are meaningless without their colours.

Whether the colour joins the digit at the perceptual or conceptual level has been object of debate (e.g., Rich \& Mattingley, 2002), and several classifications for the different subtypes of synaesthesia have been proposed. According to Ramachandran and Hubbard (2001), synaesthesia can be classified as "lower" or "higher": in the former the synaesthetic experience is driven by the perceptual experience of a stimulus, in the latter it is driven by the processing of the stimulus at the conceptual level. On the one hand, TT might be considered a "high" synaesthete, since the conceptual/semantic level is accessed only when both color and digits match the synaesthetic experience. On the other hand, the current results also indicate that synaesthetic information is already recognized at the perceptual level. Indeed, results from experiment 2 (synaesthetic colours) and 5 (shades of grey) led TT to opposite performance, whereas controls showed the darker flanker bias in both cases. The idea that both perceptual and conceptual level of processing can co-exist in one synaesthete is in line with the Integrated Model (Hubbard, 2007; see also: Hubbard, Brang, \& Ramachandran, 2011) suggesting that both colour-perception processes and binding processes (possibly at the conceptual level) are directly yet differently involved in synaesthesia. Results from experiment 2 and 5 indicate that TT processes, already at the perceptual level, colours that are associated to numbers differently than colours that are not associated to numbers (see also: McErlean \& Banissy, 2017). New lines of research should investigate to which extent luminance and synaesthetic colour are differently processed, and in which way synaesthetes tendency to associate darker colours to larger numbers (Cohen Kadosh et al., 2007b) might play a role in the development of differential processing for synaesthetic and non-synaesthetic colours.

Finally, other factors, such as the unconscious processing of stimuli (Mattingley, Rich, Yelland, \& Bradshaw, 2001) or a reduced amount of attention allocated to a synaesthetic stimulus (Rich \& Mattingley, 2002), have already been shown to limit the access to the synaesthetic experience, indicating that the enriched perceptual experience of synaesthetes is not free from constraints. A recent study by Mas-Casadesús and Gherri (2017) showed that synaesthethes are more efficient than non synaesthethes in ignoring irrelevant information in a cross-modal congruency task, suggesting that synaesthethes might benefit from enhanced attentional filtering skills. While filtering abilities plausibly play a role in the manifestation of the larger-digit bias, enhanced selective attention in synaesthesia cannot fully explain TT's performance. Indeed, if TT was simply better than controls in filtering irrelevant information, he would have shown no effect of flankers in experiment 3 and 5, which was not the case. Future research on a larger sample of synaesthetes will be useful to confirm the generalization of the conclusions raised from this study to a specific subtype of synaesthete, or to synaesthesia in general. The broad research area on synaesthesia have confirmed across many years of study that this phenomenon is highly heterogeneous, within and between synaesthetes (e.g., Cohen Kadosh et al., 2005). In this sense, single case studies confirm their usefulness to unveil informative aspects of this phenomenon (e.g., Arend, Ofir, \& Henik, 2016), aspects that might remain hidden in group studies.

In conclusion, although multisensory experiences in synaesthesia have sometimes been associated with creativity in the arts (Ward, Thompson-Lake, Ely, \& Kaminski, 2008), and accordingly might enrich a synaesthetes' perceptual life, the present study suggests that synaesthetic experience, when mediating access to semantics, might suffer under specific circumstances from rigidity. This idea is in line with a recent finding by Banissy et al. (2013) showing that graphemecolour synaesthesia can have both positive and negative effects on perceptual processing (for a discussion see also McCarthy \& Caplovitz, 2014). Consequently, this idea underlines the importance to explore how cognitive processes outside the synaesthetic experience can be positively or negatively affected in synaesthetes. An intense multisensory experience, as synaesthesia is, always has some costs.

Acknowledgements MR is supported by a grant from MIUR (Dipartimenti di Eccellenza DM 11/05/2017 n.262) to the Department of General Psychology, University of Padua. The authors thank Davide Richiello for providing us with help in data collection, Carlo Toneatto (University of Milano-Bicocca) for providing with help in the experimental programming, and Mario Bonato (University of Padua) for insightful comments. Data and materials will be available upon request. A special thanks to TT for the continued participation in these experiments.

Publisher's note Springer Nature remains neutral with regard to jurisdictional claims in published maps and institutional affiliations.

\section{References}

Arduino, L. S., Previtali, P., \& Girelli, L. (2010). The centre is not in the middle: Evidence from line and word bisection. Neuropsychologia, 48(7), 2140-2146.

Arend, I., Ofir, S., \& Henik, A. (2016). What spatial coordinate defines color-space synesthesia?. Brain and Cognition, 105, 88-94.

Baldwin, J. M. (1895). The effect of size-contrast upon judgments of position in the retinal field. Psychological Review, 2(3), 244.

Banissy, M. J., Tester, V., Muggleton, N. G., Janik, A. B., Davenport, A., Franklin, A., . . \& \& Ward, J. (2013). Synesthesia for color is linked to improved color perception but reduced motion perception. Psychological Science, 24(12), 2390-2397.

Barnett, K. J., Finucane, C., Asher, J. E., Bargary, G., Corvin, A. P., Newell, F. N., \& Mitchell, K. J. (2008). Familial patterns and the 
origins of individual differences in synaesthesia. Cognition, 106(2), 871-893.

Beeli, G., Esslen, M., \& Jäncke, L. (2007). Frequency correlates in grapheme-color synaesthesia. Psychological Science, 18(9), 788792.

Berteletti, I., Hubbard, E. M., \& Zorzi, M. (2010). Implicit versus explicit interference effects in a number-color synesthete. Cortex, 46(2), 170-177.

Bonato, M., Priftis, K., Marenzi, R., \& Zorzi, M. (2008). Modulation of hemispatial neglect by directional and numerical cues in the line bisection task. Neuropsychologia, 46(2), 426-433.

Brang, D., Edwards, L., Ramachandran, V. S., \& Coulson, S. (2008). Is the sky 2? Contextual priming in grapheme-color synaesthesia. Psychological Science, 19(5), 421-428.

Brugger, P., Knoch, D., Mohr, C., \& Gianotti, L. R. R. (2004). Is digitcolor synaesthesia strictly unidirectional? Preliminary evidence for an implicitly colored number space in three synaesthetes. Acta Neuropsychologica, 2, 252-258.

Cohen Kadosh, R., \& Henik, A. (2006). When a line is a number: Color yields magnitude information in a digit-color synesthete. Neuroscience, 137(1), 3-5.

Cohen Kadosh, R., \& Henik, A. (2007). Can synaesthesia research inform cognitive science? Trends in Cognitive Sciences, 11(4), 177-184.

Cohen Kadosh, R., Sagiv, N., Linden, D. E., Robertson, L. C., Elinger, G., \& Henik, A. (2005). When blue is larger than red: Colors influence numerical cognition in synesthesia. Journal of Cognitive Neuroscience, 17(11), 1766-1773.

Cohen Kadosh, R., Kadosh, K. C., \& Henik, A. (2007a). The neuronal correlate of bidirectional synesthesia: A combined event-related potential and functional magnetic resonance imaging study. Journal of Cognitive Neuroscience, 19(12), 2050-2059.

Cohen Kadosh, R., Henik, A., \& Walsh, V. (2007b). Small is bright and big is dark in synaesthesia. Current Biology, 17(19), R834-R835.

Crawford, J. R., \& Garthwaite, P. H. (2005). Testing for suspected impairments and dissociations in single-case studies in neuropsychology: Evaluation of alternatives using monte carlo simulations and revised tests for dissociations. Neuropsychology, 19(3), 318.

Crawford, J. R., \& Howell, D. C. (1998). Comparing an individual's test score against norms derived from small samples. The Clinical Neuropsychologist, 12(4), 482-486.

Day, S. (2005). Some demographic and socio-cultural aspects of synesthesia. In L. C. Robertson \& N. Sagiv (Eds.). Synesthesia: Perspectives from cognitive neuroscience (pp. 11-33). New York, NY: Oxford University Press.

Dehaene, S., Bossini, S., \& Giraux, P. (1993). The mental representation of parity and number magnitude. Journal of Experimental Psychology: General, 122(3), 371.

Eagleman, D. M. (2009). The objectification of overlearned sequences: A new view of spatial sequence synesthesia. Cortex, 45(10), 12661277.

Fischer, M. H. (2001). Number processing induces spatial performance biases. Neurology, 57(5), 822-826.

Galton, F. (1880). Visualised numerals. Nature, 21(533), 252-256.

Gebuis, T., Nijboer, T. C., \& Van der Smagt, M. J. (2009). Multiple dimensions in bi-directional synesthesia. European Journal of Neuroscience, 29(8), 1703-1710.

Gertner, L., Henik, A., \& Cohen Kadosh, R. (2009). When 9 is not on the right: Implications from number-form synesthesia. Consciousness and Cognition, 18(2), 366-374.

Gevers, W., Imbo, I., Cohen Kadosh, R., Fias, W., \& Hartsuiker, R.J. (2010). Bidirectionality in synesthesia: Evidence from a multiplication verification task. Experimental Psychology, 57, 178-184.

Goller, A. I., Otten, L. J., \& Ward, J. (2009). Seeing sounds and hearing colors: An event-related potential study of auditory-visual synesthesia. Journal of Cognitive Neuroscience, 21(10), 1869-1881. de Hevia, M. D., Girelli, L., \& Vallar, G. (2006). Numbers and space: A cognitive illusion? Experimental Brain Research, 168(1-2), 254264.

Hubbard, E. M. (2007). Neurophysiology of synesthesia. Current psychiatry reports, 9(3), 193-199.

Hubbard E. M., Ranzini M., Piazza M., Dehaene S. (2009). What information is critical to elicit interference in number-form synaesthesia. Cortex, 45(10), 1200-1216.

Hubbard, E. M., Brang, D., \& Ramachandran, V. S. (2011). The crossactivation theory at 10. Journal of Neuropsychology, 5(2), 152-177.

Janik McErlean, A. B., \& Banissy, M. J. (2017). Color processing in synesthesia: What synesthesia can and cannot tell us about mechanisms of color processing. Topics in Cognitive Science, 9(1), 215 227.

Johnson, A., Jepma, M., \& De Jong, R. (2007). Colours sometimes count: Awareness and bidirectionality in grapheme-colour synaesthesia. The Quarterly Journal of Experimental Psychology, 60(10), 14061422.

Knoch, D., Gianotti, L. R., Mohr, C., \& Brugger, P. (2005). Synesthesia: When colors count. Cognitive Brain Research, 25(1), 372-374.

MacLeod, C. M., \& MacDonald, P. A. (2000). Interdimensional interference in the Stroop effect: Uncovering the cognitive and neural anatomy of attention. Trends in Cognitive Sciences, 4(10), 383-391.

Mas-Casadesús, A., \& Gherri, E. (2017). Ignoring irrelevant information: enhanced intermodal attention in synaesthetes. Multisensory Research, 30(3-5), 253-277.

Mattingley, J. B., Rich, A. N., Yelland, G., \& Bradshaw, J. L. (2001). Unconscious priming eliminates automatic binding of colour and alphanumeric form in synaesthesia. Nature, 410(6828), 580-582.

McCarthy, J. D., \& Caplovitz, G. P. (2014). Color synesthesia improves color but impairs motion perception. Trends in Cognitive Sciences, 18(5), 224-226.

McCarthy, J. D., Barnes, L. N., Alvarez, B. D., \& Caplovitz, G. P. (2013). Two plus blue equals green: Grapheme-color synesthesia allows cognitive access to numerical information via color. Consciousness and Cognition, 22(4), 1384-1392.

Meier, B., \& Rothen, N. (2007). When conditioned responses "fire back": bidirectional cross-activation creates learning opportunities in synesthesia. Neuroscience, 147(3), 569-572.

Meier, B., \& Rothen, N. (2009). Training grapheme-colour associations produces a synaesthetic Stroop effect, but not a conditioned synaesthetic response. Neuropsychologia, 47(4), 1208-1211.

Mills, C. B. (1999). Digit synaesthesia: A case study using a Stroop-type test. Cognitive Neuropsychology, 16(2), 181-191.

Niccolai, V., Jennes, J., Stoerig, P., \& Van Leeuwen, T. M. (2012). Modality and variability of synesthetic experience. The American Journal of Psychology, 125(1), 81-94.

Niessen, E., Fink, G. R., Schweitzer, L., Kluender, N., \& Weiss, P. H. (2015). Implicit interactions between number and space in digitcolor synesthesia. Cortex, 64, 225-234.

Perrone, G., de Hevia, M. D., Bricolo, E., \& Girelli, L. (2010). Numbers can move our hands: a spatial representation effect in digits handwriting. Experimental Brain Research, 205(4), 479-487.

Piazza, M., Pinel, P., \& Dehaene, S. (2006). Objective correlates of an unusual subjective experience: A single-case study of number-form synaesthesia. Cognitive Neuropsychology, 23(8), 1162-1173.

Pinel, P., Piazza, M., Le Bihan, D., \& Dehaene, S. (2004). Distributed and overlapping cerebral representations of number, size, and luminance during comparative judgments. Neuron, 41(6), 983-993.

Ramachandran, V. S., \& Hubbard, E. M. (2001). Synaesthesia-a window into perception, thought and language. Journal of Consciousness Studies, 8(12), 3-34.

Ranzini, M. (2010). The influence of mathematical expertise on number processing. Ph.D. Thesis, University of Pavia, Pavia. 
Ranzini, M., \& Girelli, L. (2012). Exploiting illusory effects to disclose similarities in numerical and luminance processing. Attention, Perception, \& Psychophysics, 74(5), 1001-1008.

Rich, A. N., \& Mattingley, J. B. (2002). Anomalous perception in synaesthesia: A cognitive neuroscience perspective. Nature Reviews Neuroscience, 3(1), 43-52.

Rich, A. N., Bradshaw, J. L., \& Mattingley, J. B. (2005). A systematic, large-scale study of synaesthesia: Implications for the role of early experience in lexical-color associations. Cognition, 98(1), 53-84.

Rothen, N., Nyffeler, T., von Wartburg, R., Müri, R., \& Meier, B. (2010). Parieto-occipital suppression eliminates implicit bidirectionality in grapheme-colour synaesthesia. Neuropsychologia, 48(12), 34823487.

Sagiv, N., Simner, J., Collins, J., Butterworth, B., \& Ward, J. (2006). What is the relationship between synaesthesia and visuo-spatial number forms? Cognition, 101(1), 114-128.

Simner, J., Mulvenna, C., Sagiv, N., Tsakanikos, E., Witherby, S. A., Fraser, C., . . \& \& Ward, J. (2006). Synaesthesia: The prevalence of atypical cross-modal experiences. Perception, 35(8), 1024-1033.
Smilek, D., Carriere, J. S. A., Dixon, M. J., \& Merikle, P. M. (2007). Grapheme frequency and color luminance in grapheme-color synaesthesia. Psychological Science, 18(9), 793-795.

Stroop, J.R. (1935). Studies of interference in serial verbal reactions. Journal of Experimental Psychology, 18(6), 643-662.

Teichmann, A. L., Nieuwenstein, M. R., \& Rich, A. N. (2015). Red, green, blue equals 1, 2, 3: Digit-color synesthetes can use structured digit information to boost recall of color sequences. Cognitive Neuroscience, 6(2-3), 100-110.

Ward, J., Thompson-Lake, D., Ely, R., \& Kaminski, F. (2008). Synaesthesia, creativity and art: What is the link? British Journal of Psychology, 99(1), 127-141.

Weiss, P. H., Kalckert, A., \& Fink, G. R. (2009). Priming letters by colors: evidence for the bidirectionality of grapheme-color synesthesia. Journal of Cognitive Neuroscience, 21(10), 2019-2026.

Wollen, K. A., \& Ruggiero, F. T. (1983). Colored-letter synaesthesia. Journal of Mental Imagery, 7, 83-86. 